\title{
NEW INEQUALITIES ON LIPSCHITZ FUNCTIONS
}

\author{
İmdat İşcan, Cuma Altunsoy and Huriye Kadakal
}

(C) by University of Niš, Serbia | Creative Commons Licence: CC BY-NC-ND Abstract. In this study, some inequalities of Hermite Hadamard type obtained for $p$ convex functions are given for Lipschitz mappings. Also, some applications for special means have been given.

Keywords: Hermite Hadamard inequalities; $p$-convex functions; convex functions.

\section{Preliminaries and fundamentals}

Definition 1.1. [21] A function $f: I \subseteq \mathbb{R} \rightarrow \mathbb{R}$ is said to be convex if the inequality

$$
f(t x+(1-t) y) \leq t f(x)+(1-t) f(y)
$$

is valid for all $x, y \in I$ and $t \in[0,1]$. If this inequality reverses, then the function $f$ is said to be concave on interval $I \neq \varnothing$.

This definition is well known in literature. Convexity theory has appeared as a powerful technique to study a wide class of unrelated problems in pure and applied sciences.

One of the most important integral inequalities for convex functions is the Hermite-Hadamard inequality. The following double inequality is well known as the Hadamard inequality in literature.

Theorem 1.1. Let $f: I \subseteq \mathbb{R} \rightarrow \mathbb{R}$ be a convex function defined on the interval $I$ of real numbers and $a, b \in I$ with $a<b$. Then the inequality

$$
f\left(\frac{a+b}{2}\right) \leq \frac{1}{b-a} \int_{a}^{b} f(x) d x \leq \frac{f(a)+f(b)}{2}
$$

is known as the Hermite-Hadamard inequality.

Received October 23, 2018; accepted May 05, 2020

2010 Mathematics Subject Classification. Primary 26A51; Secondary 26D10, 26D15 
Some refinements of the Hermite-Hadamard inequality on convex functions have been extensively investigated by a number of authors (e.g., [2, 3, 4, 11, 17, 20, 23, 24]).

Let us recall some definitions of several kinds of convex functions:

Definition 1.2. [5] Let $I \subset \mathbb{R} \backslash\{0\}$ be a real interval. A function $f: I \rightarrow \mathbb{R}$ is said to be harmonically convex, if

$$
f\left(\frac{x y}{t x+(1-t) y}\right) \leq t f(y)+(1-t) f(x)
$$

for all $x, y \in I$ and $t \in[0,1]$. If this inequality is reversed, then the function $f$ is said to be harmonically concave.

Definition 1.3. [7] Let $I \subset(0, \infty)$ be a real interval and $p \in \mathbb{R} \backslash\{0\}$. A function $f: I \rightarrow \mathbb{R}$ is said to be a $p$-convex function, if

$$
f\left(\left[t x^{p}+(1-t) y^{p}\right]^{\frac{1}{p}}\right) \leq t f(x)+(1-t) f(y)
$$

for all $x, y \in I$ and $t \in[0,1]$. If this inequality is reversed, then the function $f$ is said to be $p$-concave.

According to this definition, it can be easily seen that for $p=1$ and $p=-1$, $p$-convexity is reduced to ordinary convexity and harmonical convexity of functions defined on $I \subset(0, \infty)$, respectively.

Hermite-Hadamard inequalities for the $p$-convex function are the following:

Theorem 1.2. [7] Let $f: I \subset(0, \infty) \rightarrow \mathbb{R}$ be a p-convex function, $p \in \mathbb{R} \backslash\{0\}$ and $a, b \in I$ with $a<b$. If $f \in L[a, b]$ then we have

$$
f\left(\left[\frac{a^{p}+b^{p}}{2}\right]^{\frac{1}{p}}\right) \leq \frac{p}{b^{p}-a^{p}} \int_{a}^{b} \frac{f(x)}{x^{1-p}} d x \leq \frac{f(a)+f(b)}{2} .
$$

These inequalities are sharp [7]. We refer the reader to the recent papers related to $p$-convexity (see $[13,14,15,16,18,19])$ and references therein.

The purpose of this article is to obtain new inequalities on the right and left sides of the inequality (1.2) for Lipshitz functions.

Definition 1.4. [22] $f: I \rightarrow \mathbb{R}$ is said to satisfy the Lipschitz condition if there is a constant $M>0$ such that

$$
|f(x)-f(y)| \leq M|x-y|, \quad \forall x, y \in I .
$$

Theorem 1.3. [22] If $f: I \rightarrow \mathbb{R}$ is convex, then $f$ satisfies a Lipschitz condition on any closed interval $[a, b]$ contained in the interior $I^{\circ}$ of $I$. Consequently, $f$ is absolutely continuous on $[a, b]$ and continuous on $I^{\circ}$. 
Lemma 1.1. [1] Let the function $f: I \subset \mathbb{R} \rightarrow \mathbb{R}$ a differentiable function on interval $I, a, b \in I$ with $a<b$ and $M=\sup _{t \in[a, b]}\left|f^{\prime}(t)\right|<\infty$. Then the function $f$ is an M-Lipschitzian functions.

In [1], Dragomir et al. obtained new inequalities on the right and left sides of the inequality (1.1) for Lipshitz functions as follows.

Theorem 1.4. [1] Let $f: I \subset \mathbb{R} \rightarrow \mathbb{R}$ be an $M$-Lipschitzian mapping on $I$ and $a, b \in I$ with $a<b$. Then we have the inequalities:

$$
\left|f\left(\frac{a+b}{2}\right)-\frac{1}{b-a} \int_{a}^{b} f(x) d x\right| \leq \frac{M}{4}(b-a)
$$

and

$$
\left|\frac{f(a)+f(b)}{2}-\frac{1}{b-a} \int_{a}^{b} f(x) d x\right| \leq \frac{M}{3}(b-a) .
$$

Corollary 1.1. [1]Let $f: I \subset(0, \infty) \rightarrow \mathbb{R}$ be a convex and differentiable function on interval $I$ and $a, b \in I$ with $a<b$ and $M:=\sup _{t \in[a, b]}\left|f^{\prime}(x)\right|<\infty$. Then we have the inequalities:

$$
0 \leq \frac{1}{b-a} \int_{a}^{b} f(x) d x-f\left(\frac{a+b}{2}\right) \leq \frac{M}{4}(b-a)
$$

and

$$
0 \leq \frac{f(a)+f(b)}{2}-\frac{1}{b-a} \int_{a}^{b} f(x) d x \leq \frac{M}{3}(b-a) .
$$

Some results obtained in this study are reduced to the results of Theorem 1.4 and Corollary 1.1 in special cases. For more recent results connected with inequalities of the Hermite-Hadamard type on Lipschitzian functions, see $[6,8,9,10]$.

In the following part, we will give some necessary definitions and simple mathematical inequalities that will be used to achieve our main results.

Definition 1.5. [12] The beta function denoted by $\beta(m, n)$ is defined as

$$
\beta(m, n)=\int_{0}^{1} x^{m-1}(1-x)^{n-1} d x .
$$

Definition 1.6. [12] The hypergeometric function denoted by ${ }_{2} F_{1}(a, b ; c ; z)$ is defined by the integral equality

$$
{ }_{2} F_{1}(a, b ; c ; z)=\frac{1}{\beta(b, c-b)} \int_{0}^{1} t^{b-1}(1-t)^{c-b-1}(1-z t)^{-a} d t, c>b>0,|z|<1 .
$$


The following Lemmas are well known in literature. Especially, Lemma 1.3 and Lemma 1.4 are easily seen from the Lagrange mean value theorem.

Lemma 1.2. Let $0 \leq x<y$. Then the following inequality holds for $0<\alpha \leq 1$ :

$$
\left|y^{\alpha}-x^{\alpha}\right| \leq|y-x|^{\alpha}
$$

Lemma 1.3. Let $0<x<y$. Then the following inequality holds for $\alpha<0$ :

$$
\left|y^{\alpha}-x^{\alpha}\right| \leq|y-x|(-\alpha) x^{\alpha-1}
$$

Lemma 1.4. Let $0<x<y$. Then the following inequality holds for $\alpha \geq 1$ :

$$
\left|y^{\alpha}-x^{\alpha}\right| \leq|y-x| \alpha y^{\alpha-1}
$$

Let $0<x<y$, throughout this paper we will use

$$
\begin{aligned}
A & =A(x, y)=\frac{x+y}{2} \\
G & =G(x, y)=\sqrt{x y} \\
H & =H(x, y)=\frac{2 x y}{x+y} \\
M_{p} & =M_{p}(x, y)=\left(\frac{x^{p}+y^{p}}{2}\right)^{\frac{1}{p}} \\
I & =I(x, y)=\frac{1}{e}\left(\frac{y^{y}}{x^{x}}\right)^{\frac{1}{y-x}} \\
L & =L(x, y)=\frac{x-y}{\ln x-\ln y} \\
L_{p} & =L_{p}(x, y)=\left(\frac{y^{p+1}-x^{p+1}}{(p+1)(y-x)}\right)^{\frac{1}{p}}, p \neq-1,0
\end{aligned}
$$

for the arithmetic, geometric, harmonic, power mean of the order $p$, identric, logarithmic and $p$-logarithmic mean, respectively.

\section{Main results}

In this section, we shall establish some Hermite-Hadamard-type inequalities for Lipschitzian functions.

Theorem 2.1. $f: I \subset(0, \infty) \rightarrow \mathbb{R}$ be an $M$-Lipschitzian function on the interval $I$ of real numbers and $a, b \in I$ with $a<b$. Then following inequalities hold: 
a) For $p \geq 1$;

\begin{tabular}{l|l} 
i) & $\left|\frac{f(a)+f(b)}{2}-\frac{p}{b^{p}-a^{p}} \int_{a}^{b} \frac{f(x)}{x^{1-p}} d x\right| \leq 2 M\left|b^{p}-a^{p}\right|^{\frac{1}{p}} \frac{p^{2}}{(p+1)(2 p+1)}$ \\
ii) & $\left|\frac{p}{b^{p}-a^{p}} \int_{a}^{b} \frac{f(x)}{x^{1-p}} d x-f\left(\left[\frac{a^{p}+b^{p}}{2}\right]^{\frac{1}{p}}\right)\right| \leq M\left(\frac{1}{2}\right)^{\frac{1}{p}}\left|b^{p}-a^{p}\right|^{\frac{1}{p}} \frac{p}{p+1}$.
\end{tabular}

b) For $p<0$;

i) $\quad\left|\frac{f(a)+f(b)}{2}-\frac{p}{b^{p}-a^{p}} \int_{a}^{b} \frac{f(x)}{x^{1-p}} d x\right|$ $\leq M \frac{b^{p}-a^{p}}{6 p b^{p-1}}\left[{ }_{2} F_{1}\left(1-\frac{1}{p}, 2 ; 4 ; 1-\left(\frac{b}{a}\right)^{p}\right)+1\right]$,

ii) $\quad\left|\frac{p}{b^{p}-a^{p}} \int_{a}^{b} \frac{f(x)}{x^{1-p}} d x-f\left(\left[\frac{a^{p}+b^{p}}{2}\right]^{\frac{1}{p}}\right)\right|$ $\leq M \frac{b^{p}-a^{p}}{2 p\left(\frac{a^{p}+b^{p}}{2}\right)^{1-\frac{1}{p}}}\left[{ }_{2} F_{1}\left(1-\frac{1}{p}, 1 ; 3 ; 1-\left(\frac{b}{a}\right)^{p}\right)+1\right]$

c) For $0<p<1$;

$$
\begin{gathered}
\text { i) }\left|\frac{f(a)+f(b)}{2}-\frac{p}{b^{p}-a^{p}} \int_{a}^{b} \frac{f(x)}{x^{1-p}} d x\right| \leq M \frac{b^{p}-a^{p}}{6 p}\left[a^{1-p}+b^{1-p}\right], \\
\text { ii) }\left|\frac{p}{b^{p}-a^{p}} \int_{a}^{b} \frac{f(x)}{x^{1-p}} d x-f\left(\left[\frac{a^{p}+b^{p}}{2}\right]^{\frac{1}{p}}\right)\right| \\
\leq M \frac{b^{p}-a^{p}}{4 p\left(\frac{a^{p}+b^{p}}{2}\right)^{1-\frac{1}{p}} \cdot 2} F_{1}\left(1-\frac{1}{p}, 2 ; 3 ; \frac{a^{p}-b^{p}}{a^{p}+b^{p}}\right) .
\end{gathered}
$$

Proof. a) For $p \geq 1$ :

(i) Using Lemma 1.2 and taking into account that $f$ is an $M$-Lipschitzian function on interval $I$, we have

$$
\begin{aligned}
& \left|t f(a)+(1-t) f(b)-f\left(\left[t a^{p}+(1-t) b^{p}\right]^{\frac{1}{p}}\right)\right| \\
= & \left|t\left(f(a)-f\left(\left[t a^{p}+(1-t) b^{p}\right]^{\frac{1}{p}}\right)\right)+(1-t)\left(f(b)-f\left(\left[t a^{p}+(1-t) b^{p}\right]^{\frac{1}{p}}\right)\right)\right| \\
\leq & t\left|f(a)-f\left(\left[t a^{p}+(1-t) b^{p}\right]^{\frac{1}{p}}\right)\right|+(1-t)\left|f(b)-f\left(\left[t a^{p}+(1-t) b^{p}\right]^{\frac{1}{p}}\right)\right| \\
\leq & t M\left|a-\left(t a^{p}+(1-t) b^{p}\right)^{\frac{1}{p}}\right|+(1-t) M\left|b-\left(t a^{p}+(1-t) b^{p}\right)^{\frac{1}{p}}\right| \\
\leq & M\left[t\left|\left(a^{p}\right)^{\frac{1}{p}}-\left(t a^{p}+(1-t) b^{p}\right)^{\frac{1}{p}}\right|+(1-t)\left|\left(b^{p}\right)^{\frac{1}{p}}-\left(t a^{p}+(1-t) b^{p}\right)^{\frac{1}{p}}\right|\right] \\
= & M\left[t\left|(1-t)\left(b^{p}-a^{p}\right)\right|^{\frac{1}{p}}+(1-t)\left|t\left(b^{p}-a^{p}\right)\right|^{\frac{1}{p}}\right] \\
= & M\left[t(1-t)^{\frac{1}{p}}\left|b^{p}-a^{p}\right|^{\frac{1}{p}}+(1-t) t^{\frac{1}{p}}\left|b^{p}-a^{p}\right|^{\frac{1}{p}}\right] .
\end{aligned}
$$

So, for all $t \in[0,1]$, we can write the following inequality:

$$
\begin{aligned}
& \left|t f(a)+(1-t) f(b)-f\left(\left[t a^{p}+(1-t) b^{p}\right]^{\frac{1}{p}}\right)\right| \\
\leq & M\left[t(1-t)^{\frac{1}{p}}\left|b^{p}-a^{p}\right|^{\frac{1}{p}}+(1-t) t^{\frac{1}{p}}\left|b^{p}-a^{p}\right|^{\frac{1}{p}}\right] .
\end{aligned}
$$


Integrating this inequality on $[0,1]$ over $t$ we get the inequality:

$$
\begin{aligned}
& \left|f(a) \int_{0}^{1} t d t+f(b) \int_{0}^{1}(1-t) d t-\int_{0}^{1} f\left(\left[t a^{p}+(1-t) b^{p}\right]^{\frac{1}{p}}\right) d t\right| \\
\leq & M\left|b^{p}-a^{p}\right|^{\frac{1}{p}}\left[\int_{0}^{1} t(1-t)^{\frac{1}{p}} d t+\int_{0}^{1} t^{\frac{1}{p}}(1-t) d t\right] .
\end{aligned}
$$

Here, it is easy to see that

$$
\begin{gathered}
\int_{0}^{1} t^{\frac{1}{p}}(1-t) d t=\int_{0}^{1} t(1-t)^{\frac{1}{p}} d t d t=\frac{p^{2}}{(p+1)(2 p+1)} \\
f(a) \int_{0}^{1} t d t+f(b) \int_{0}^{1}(1-t) d t=\frac{f(a)+f(b)}{2}
\end{gathered}
$$

and

$$
\int_{0}^{1} f\left(\left[t a^{p}+(1-t) b^{p}\right]^{\frac{1}{p}}\right) d t=\frac{p}{b^{p}-a^{p}} \int_{a}^{b} \frac{f(x)}{x^{p-1}} d x .
$$

Using the inequalities (2.3), (2.4) and (2.5) in the inequality (2.2), we can derive the desired inequality:

$$
\left|\frac{f(a)+f(b)}{2}-\frac{p}{b^{p}-a^{p}} \int_{a}^{b} \frac{f(x)}{x^{1-p}} d x\right| \leq 2 M\left|b^{p}-a^{p}\right|^{\frac{1}{p}} \frac{p^{2}}{(p+1)(2 p+1)}
$$

(ii) Putting $t=\frac{1}{2}$ in the inequality (2.1), we have

$$
\left|\frac{f(a)+f(b)}{2}-f\left(\left[\frac{a^{p}+b^{p}}{2}\right]^{\frac{1}{p}}\right)\right| \leq M\left(\frac{1}{2}\right)^{\frac{1}{p}}\left|b^{p}-a^{p}\right|^{\frac{1}{p}} .
$$

If we replace $a$ by $\left(t a^{p}+(1-t) b^{p}\right)^{\frac{1}{p}}$ and $b$ by $\left((1-t) a^{p}+t b^{p}\right)^{\frac{1}{p}}$ in the inequality (2.6), we obtain

$$
\begin{aligned}
& \left|\frac{f\left(\left[t a^{p}+(1-t) b^{p}\right]^{\frac{1}{p}}\right)+f\left(\left[(1-t) a^{p}+t b^{p}\right]^{\frac{1}{p}}\right)}{2}-f\left(\left[\frac{a^{p}+b^{p}}{2}\right]^{\frac{1}{p}}\right)\right| \\
\leq & M\left(\frac{1}{2}\right)^{\frac{1}{p}}\left|(2 t-1)\left(b^{p}-a^{p}\right)\right|^{\frac{1}{p}} \\
= & M\left(\frac{1}{2}\right)^{\frac{1}{p}}|2 t-1|^{\frac{1}{p}}\left|b^{p}-a^{p}\right|^{\frac{1}{p}}
\end{aligned}
$$

all $t \in[0,1]$. Now, if we integrate the inequality $(2.7)$ on $[0,1]$ over $t$ we can state that

$$
\left|\int_{0}^{1} \frac{f\left(\left[t a^{p}+(1-t) b^{p}\right]^{\frac{1}{p}}\right)}{2} d t+\int_{0}^{1} \frac{f\left(\left[(1-t) a^{p}+t b^{p}\right]^{\frac{1}{p}}\right)}{2} d t-f\left(\left[\frac{a^{p}+b^{p}}{2}\right]^{\frac{1}{p}}\right)\right|
$$




$$
\leq M\left(\frac{1}{2}\right)^{\frac{1}{p}}\left|b^{p}-a^{p}\right|^{\frac{1}{p}} \int_{0}^{1 \frac{1}{p}}|2 t-1|^{\frac{1}{p}} d t .
$$

Here, it is easy to see that

$$
\begin{aligned}
& \int_{0}^{1} f\left(\left[t a^{p}+(1-t) b^{p}\right]^{\frac{1}{p}}\right) d t=\int_{0}^{1} f\left(\left[(1-t) a^{p}+t b^{p}\right]^{\frac{1}{p}}\right) d t=\frac{p}{b^{p}-a^{p}} \int_{a}^{b} \frac{f(x)}{x^{1-p}} d x \\
& \text { and } \\
& \qquad \begin{array}{l}
(2.10) \\
\qquad \int_{0}^{1}|2 t-1|^{\frac{1}{p}} d t=\frac{p}{p+1} .
\end{array}
\end{aligned}
$$

If we put the equalities (2.9) and (2.10) in the inequality (2.8), then we have the desired inequality:

$$
\left|\frac{p}{b^{p}-a^{p}} \int_{a}^{b} \frac{f(x)}{x^{1-p}} d x-f\left(\left[\frac{a^{p}+b^{p}}{2}\right]^{\frac{1}{p}}\right)\right| \leq M\left(\frac{1}{2}\right)^{\frac{1}{p}}\left|b^{p}-a^{p}\right|^{\frac{1}{p}} \frac{p}{p+1} .
$$

b) For $p<0$ :

(i) Taking into account that $f$ is an $M$-Lipschitzian function on interval $I$, we have

$$
\begin{aligned}
& (2.11)\left|t f(a)+(1-t) f(b)-f\left(\left[t a^{p}+(1-t) b^{p}\right]^{\frac{1}{p}}\right)\right| \\
& =\left|t\left(f(a)-f\left(\left[t a^{p}+(1-t) b^{p}\right]^{\frac{1}{p}}\right)\right)+(1-t)\left(f(b)-f\left(\left[t a^{p}+(1-t) b^{p}\right]^{\frac{1}{p}}\right)\right)\right| \\
& \leq t\left|f(a)-f\left(\left[t a^{p}+(1-t) b^{p}\right]^{\frac{1}{p}}\right)\right|+(1-t)\left|f(b)-f\left(\left[t a^{p}+(1-t) b^{p}\right]^{\frac{1}{p}}\right)\right| \\
& \leq t M\left|\left(a^{p}\right)^{\frac{1}{p}}-\left(t a^{p}+(1-t) b^{p}\right)^{\frac{1}{p}}\right|+(1-t) M\left|\left(b^{p}\right)^{\frac{1}{p}}-\left(t a^{p}+(1-t) b^{p}\right)^{\frac{1}{p}}\right| .
\end{aligned}
$$

Also, using Lemma 1.3, we get

$$
\begin{aligned}
& \left|\left(a^{p}\right)^{\frac{1}{p}}-\left(t a^{p}+(1-t) b^{p}\right)^{\frac{1}{p}}\right| \\
\leq & \left|a^{p}-t a^{p}-b^{p}+t b^{p}\right|\left(-\frac{1}{p}\right)\left(t a^{p}+(1-t) b^{p}\right)^{\frac{1}{p}-1} \\
= & \left|(1-t) a^{p}-(1-t) b^{p}\right|\left(-\frac{1}{p}\right)\left(t a^{p}+(1-t) b^{p}\right)^{\frac{1}{p}-1} \\
= & (1-t) \frac{b^{p}-a^{p}}{p}\left(t a^{p}+(1-t) b^{p}\right)^{\frac{1}{p}-1}
\end{aligned}
$$

and

$$
\begin{aligned}
(2.13)\left|\left(b^{p}\right)^{\frac{1}{p}}-\left(t a^{p}+(1-t) b^{p}\right)^{\frac{1}{p}}\right| & \leq\left|b^{p}-t a^{p}-b^{p}+t b^{p}\right|\left(-\frac{1}{p}\right)\left(b^{p}\right)^{\frac{1}{p}-1} \\
& =\left|t\left(a^{p}-b^{p}\right)\right|\left(-\frac{1}{p}\right)\left(b^{p}\right)^{\frac{1}{p}-1} \\
& =t \frac{b^{p}-a^{p}}{p} b^{1-p} .
\end{aligned}
$$


If we put the equalities (2.12) and (2.13) in the inequality (2.11), we obtain the following inequality:

$$
\begin{aligned}
& \left|t f(a)+(1-t) f(b)-f\left(\left[t a^{p}+(1-t) b^{p}\right]^{\frac{1}{p}}\right)\right| \\
\leq & M \frac{b^{p}-a^{p}}{p}\left[\frac{t(1-t)}{\left(t a^{p}+(1-t) b^{p}\right)^{1-\frac{1}{p}}}+t(1-t) b^{1-p}\right] .
\end{aligned}
$$

If we integrate the inequality $(2.13)$ on $[0,1]$ over $t$ we get

$$
\begin{aligned}
& \left|f(a) \int_{0}^{1} t d t+f(b) \int_{0}^{1}(1-t) d t-\int_{0}^{1} f\left(\left[t a^{p}+(1-t) b^{p}\right]^{\frac{1}{p}}\right) d t\right| \\
\leq & M \frac{b^{p}-a^{p}}{p}\left[\int_{0}^{1} \frac{t(1-t)}{\left(t a^{p}+(1-t) b^{p}\right)^{1-\frac{1}{p}}} d t+b^{1-p} \int_{0}^{1} t(1-t) d t\right]
\end{aligned}
$$

for all $t \in[0,1]$. Here, we can write the following equality:

$$
\int_{0}^{1} \frac{t(1-t)}{\left(t a^{p}+(1-t) b^{p}\right)^{1-\frac{1}{p}}} d t=\int_{0}^{1} \frac{t(1-t)}{b^{p-1}\left[1-t\left(1-\left(\frac{a}{b}\right)^{p}\right)\right]^{1-\frac{1}{p}}} d t
$$

Further, if we calculate the equality (2.16) using the definitions of the beta and hypergeometric functions, then we obtain

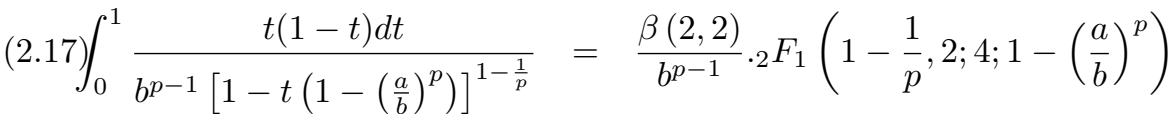

$$
\begin{aligned}
& =\frac{1}{6 b^{p-1}} \cdot 2 F_{1}\left(1-\frac{1}{p}, 2 ; 4 ; 1-\left(\frac{a}{b}\right)^{p}\right)
\end{aligned}
$$

where

$$
\beta(2,2)=\int_{0}^{1} t(1-t) d t=\frac{1}{6} .
$$

If we put the equalities (2.5) and (2.17) in the inequality (2.15), then we have the desired inequality as follows:

$$
\begin{aligned}
& \left|\frac{f(a)+f(b)}{2}-\frac{p}{b^{p}-a^{p}} \int_{a}^{b} \frac{f(x)}{x^{1-p}} d x\right| \\
\leq & M \frac{b^{p}-a^{p}}{6 p b^{p-1}}\left[{ }_{2} F_{1}\left(1-\frac{1}{p}, 2 ; 4 ; 1-\left(\frac{b}{a}\right)^{p}\right)+1\right]
\end{aligned}
$$

(ii) If we take $t=\frac{1}{2}$ and $\frac{b^{p}-a^{p}}{p}=\frac{\left|b^{p}-a^{p}\right|}{-p}$ in the inequality (2.14), we have (2.18) $\left|\frac{f(a)+f(b)}{2}-f\left(\left[\frac{a^{p}+b^{p}}{2}\right]^{\frac{1}{p}}\right)\right| \leq M \frac{\left|b^{p}-a^{p}\right|}{-4 p}\left[\frac{1}{\left(\frac{a^{p}+b^{p}}{2}\right)^{1-\frac{1}{p}}}+b^{1-p}\right]$. 
If we replace in the inequality $(2.18) a$ with $\left(t a^{p}+(1-t) b^{p}\right)^{\frac{1}{p}}$ and $b$ with $\left((1-t) a^{p}+t b^{p}\right)^{\frac{1}{p}}$, we can write the following inequality for all $t \in[0,1]$ :

$$
\begin{aligned}
& \left|\frac{f\left(\left[t a^{p}+(1-t) b^{p}\right]^{\frac{1}{p}}\right)+f\left(\left[(1-t) a^{p}+t b^{p}\right]^{\frac{1}{p}}\right)}{2}-f\left(\left[\frac{a^{p}+b^{p}}{2}\right]^{\frac{1}{p}}\right)\right| \\
\leq & M\left|\frac{(1-t) a^{p}+t b^{p}-\left(t a^{p}+(1-t) b^{p}\right)}{-4 p}\right| \\
& \times\left[\frac{1}{\left(\frac{a^{p}+b^{p}}{2}\right)^{1-\frac{1}{p}}}+\frac{1}{\left(\left((1-t) a^{p}+t b^{p}\right)^{\frac{1}{p}}\right)^{p-1}}\right] \\
= & M|2 t-1| \frac{b^{p}-a^{p}}{4 p}\left[\frac{1}{\left(\frac{a^{p}+b^{p}}{2}\right)^{1-\frac{1}{p}}}+\frac{1}{\left((1-t) a^{p}+t b^{p}\right)^{1-\frac{1}{p}}}\right]
\end{aligned}
$$

Integrating the last inequality $(2.19)$ on $[0,1]$ over $t$ we get

$$
\begin{aligned}
& \left|\frac{f\left(\left[t a^{p}+(1-t) b^{p}\right]^{\frac{1}{p}}\right)+f\left(\left[(1-t) a^{p}+t b^{p}\right]^{\frac{1}{p}}\right)}{2}-f\left(\left[\frac{a^{p}+b^{p}}{2}\right]^{\frac{1}{p}}\right)\right| \\
\leq & M \frac{b^{p}-a^{p}}{4 p}\left[\frac{1}{\left(\frac{a^{p}+b^{p}}{2}\right)^{1-\frac{1}{p}}} \int_{0}^{1}|2 t-1| d t+\int_{0}^{1} \frac{|2 t-1|}{\left((1-t) a^{p}+t b^{p}\right)^{1-\frac{1}{p}}} d t\right]
\end{aligned}
$$

where

$$
\int_{0}^{1}|2 t-1| d t=\frac{1}{2}
$$

Now, let calculate the second integral in the inequality (2.22):

$$
\begin{aligned}
& \int_{0}^{1} \frac{|2 t-1|}{\left((1-t) a^{p}+t b^{p}\right)^{1-\frac{1}{p}}} d t \\
= & \int_{0}^{\frac{1}{2}} \frac{1-2 t}{\left((1-t) a^{p}+t b^{p}\right)^{1-\frac{1}{p}}} d t+\int_{\frac{1}{2}}^{1} \frac{2 t-1}{\left((1-t) a^{p}+t b^{p}\right)^{1-\frac{1}{p}}} d t .
\end{aligned}
$$

It is easy to see that

$$
\begin{aligned}
(2.23) \int_{0}^{\frac{1}{2}} \frac{(1-2 t) d t}{\left((1-t) a^{p}+t b^{p}\right)^{1-\frac{1}{p}}} & =\frac{\beta(2,1)}{2\left(\frac{a^{p}+b^{p}}{2}\right)^{1-\frac{1}{p}} \cdot 2} F_{1}\left(1-\frac{1}{p}, 2 ; 3 ; \frac{a^{p}-b^{p}}{a^{p}+b^{p}}\right) \\
& =\frac{1}{4\left(\frac{a^{p}+b^{p}}{2}\right)^{1-\frac{1}{p}} \cdot 2} F_{1}\left(1-\frac{1}{p}, 2 ; 3 ; \frac{a^{p}-b^{p}}{a^{p}+b^{p}}\right)
\end{aligned}
$$

and

$$
(2.24) \int_{\frac{1}{2}}^{1} \frac{2 t-1}{\left((1-t) a^{p}+t b^{p}\right)^{1-\frac{1}{p}}} d t=\frac{1}{4\left(\frac{a^{p}+b^{p}}{2}\right)^{1-\frac{1}{p}} \cdot 2} F_{1}\left(1-\frac{1}{p}, 2 ; 3 ; \frac{a^{p}-b^{p}}{a^{p}+b^{p}}\right)
$$


where

$$
\beta(2,1)=\int_{0}^{1} t d t=\frac{1}{2}
$$

Adding the equalities (2.23) and (2.24), we get

$(2.25) \int_{0}^{1} \frac{|2 t-1|}{\left((1-t) a^{p}+t b^{p}\right)^{1-\frac{1}{p}}} d t=\frac{1}{2\left(\frac{a^{p}+b^{p}}{2}\right)^{1-\frac{1}{p}}} \cdot{ }_{2} F_{1}\left(1-\frac{1}{p}, 2 ; 3 ; \frac{a^{p}-b^{p}}{a^{p}+b^{p}}\right)$.

If we put the equalities (2.21) and (2.25) in the inequality (2.20), then we obtain the following inequality for all $t \in[0,1]$ :

$$
\begin{aligned}
& \left|\frac{p}{b^{p}-a^{p}} \int_{a}^{b} \frac{f(x)}{x^{1-p}} d x-f\left(\left[\frac{a^{p}+b^{p}}{2}\right]^{\frac{1}{p}}\right)\right| \\
\leq & M \frac{b^{p}-a^{p}}{2 p\left(\frac{a^{p}+b^{p}}{2}\right)^{1-\frac{1}{p}}}\left[{ }_{2} F_{1}\left(1-\frac{1}{p}, 1 ; 3 ; 1-\left(\frac{b}{a}\right)^{p}\right)+1\right] .
\end{aligned}
$$

c) For $0<p<1$

Using the inequality (2.11), we can write the inequality

$$
\begin{aligned}
& \quad\left|t f(a)+(1-t) f(b)-f\left(\left[t a^{p}+(1-t) b^{p}\right]^{\frac{1}{p}}\right)\right| \\
& \leq \quad t M\left|\left(a^{p}\right)^{\frac{1}{p}}-\left(t a^{p}+(1-t) b^{p}\right)^{\frac{1}{p}}\right|+(1-t) M\left|\left(b^{p}\right)^{\frac{1}{p}}-\left(t a^{p}+(1-t) b^{p}\right)^{\frac{1}{p}}\right|
\end{aligned}
$$

for all $t \in[0,1]$. Also, by using Lemma 1.4, we get

$$
\begin{aligned}
\left|\left(a^{p}\right)^{\frac{1}{p}}-\left(t a^{p}+(1-t) b^{p}\right)^{\frac{1}{p}}\right| & \leq\left|a^{p}-t a^{p}-b^{p}+t b^{p}\right|\left(\frac{1}{p}\right)\left(a^{p}\right)^{\frac{1}{p}-1} \\
& =(1-t) \frac{b^{p}-a^{p}}{p} a^{1-p}
\end{aligned}
$$

and

$$
\begin{aligned}
\left|\left(b^{p}\right)^{\frac{1}{p}}-\left(t a^{p}+(1-t) b^{p}\right)^{\frac{1}{p}}\right| & \leq\left|b^{p}-t a^{p}-b^{p}+t b^{p}\right|\left(\frac{1}{p}\right)\left(b^{p}\right)^{\frac{1}{p}-1} \\
& =t \frac{b^{p}-a^{p}}{p} b^{1-p} .
\end{aligned}
$$

From the inequalities (2.27) and (2.28), the inequality (2.26) can be wriiten as

$$
\begin{aligned}
& \left|t f(a)+(1-t) f(b)-f\left(\left[t a^{p}+(1-t) b^{p}\right]^{\frac{1}{p}}\right)\right| \\
\leq & M \frac{b^{p}-a^{p}}{p}\left[t(1-t) a^{1-p}+t(1-t) b^{1-p}\right] .
\end{aligned}
$$


Integrating the inequality $(2.29)$ on $[0,1]$ over $t$ we obtain

$$
\begin{aligned}
& \left|f(a) \int_{0}^{1} t d t+f(b) \int_{0}^{1}(1-t) d t-\int_{0}^{1} f\left(\left[t a^{p}+(1-t) b^{p}\right]^{\frac{1}{p}}\right) d t\right| \\
\leq & M \frac{b^{p}-a^{p}}{p}\left[a^{1-p} \int_{0}^{1} t(1-t) d t+b^{1-p} \int_{0}^{1} t(1-t) d t\right]
\end{aligned}
$$

where

$$
\int_{0}^{1} t(1-t) d t=\frac{1}{6}
$$

If we put $(2.4),(2.5)$ and $(2.31)$ in the inequality $(2.29)$, then we get the following inequality for all $t \in[0,1]$ :

$$
\left|\frac{f(a)+f(b)}{2}-\frac{p}{b^{p}-a^{p}} \int_{a}^{b} \frac{f(x)}{x^{1-p}} d x\right| \leq M \frac{\left|b^{p}-a^{p}\right|}{6 p}\left[a^{1-p}+b^{1-p}\right] .
$$

Putting $t=\frac{1}{2}$ in the inequality (2.29), we have

$$
\left|\frac{f(a)+f(b)}{2}-f\left(\left[\frac{a^{p}+b^{p}}{2}\right]^{\frac{1}{p}}\right)\right| \leq M \frac{\left|b^{p}-a^{p}\right|}{4 p}\left[a^{1-p}+b^{1-p}\right] .
$$

If we replace in the inequality $(2.33) a$ with $\left(t a^{p}+(1-t) b^{p}\right)^{\frac{1}{p}}$ and $b$ with $\left((1-t) a^{p}+t b^{p}\right)^{\frac{1}{p}}$, we can write the following inequality for all $t \in[0,1]$ :

$$
\begin{aligned}
& \left|\frac{f\left(\left[t a^{p}+(1-t) b^{p}\right]^{\frac{1}{p}}\right)+f\left(\left[(1-t) a^{p}+t b^{p}\right]^{\frac{1}{p}}\right)}{2}-f\left(\left[\frac{a^{p}+b^{p}}{2}\right]^{\frac{1}{p}}\right)\right| \\
\leq & M \frac{\left|\left(\left[(1-t) a^{p}+t b^{p}\right]^{\frac{1}{p}}\right)^{p}-\left(\left[t a^{p}+(1-t) b^{p}\right]^{\frac{1}{p}}\right)^{p}\right|}{4 p} \\
& \times\left[\left(\left[t a^{p}+(1-t) b^{p}\right]^{\frac{1}{p}}\right)^{1-p}+\left(\left[(1-t) a^{p}+t b^{p}\right]^{\frac{1}{p}}\right)^{1-p}\right] \\
= & M \frac{b^{p}-a^{p}}{4 p}\left[\frac{|2 t-1|}{\left(t a^{p}+(1-t) b^{p}\right)^{1-\frac{1}{p}}}+\frac{|2 t-1|}{\left((1-t) a^{p}+t b^{p}\right)^{1-\frac{1}{p}}}\right] .
\end{aligned}
$$

Integrating the inequality $(2.34)$ on the interval $[0,1]$ over $t$ we have

$$
\begin{aligned}
& \left|\int_{0}^{1} \frac{f\left(\left[t a^{p}+(1-t) b^{p}\right]^{\frac{1}{p}}\right)}{2} d t+\int_{0}^{1} \frac{f\left(\left[(1-t) a^{p}+t b^{p}\right]^{\frac{1}{p}}\right)}{2} d t-f\left(\left[\frac{a^{p}+b^{p}}{2}\right]^{\frac{1}{p}}\right)\right| \\
& (2.35) \leq M \frac{b^{p}-a^{p}}{4 p}\left[\int_{0}^{1} \frac{|2 t-1|}{\left(t a^{p}+(1-t) b^{p}\right)^{1-\frac{1}{p}}} d t+\int_{0}^{1} \frac{|2 t-1|}{\left((1-t) a^{p}+t b^{p}\right)^{1-\frac{1}{p}}} d t\right] .
\end{aligned}
$$


Here, using (2.22) we see that the following equalities

$$
\begin{gathered}
\int_{0}^{1} \frac{|2 t-1|}{\left(t a^{p}+(1-t) b^{p}\right)^{1-\frac{1}{p}}} d t=\int_{0}^{1} \frac{|2 t-1|}{\left((1-t) a^{p}+t b^{p}\right)^{1-\frac{1}{p}}} d t \\
=\frac{1}{2\left(\frac{a^{p}+b^{p}}{2}\right)^{1-\frac{1}{p}} \cdot 2} F_{1}\left(1-\frac{1}{p}, 2 ; 3 ; \frac{a^{p}-b^{p}}{a^{p}+b^{p}}\right)
\end{gathered}
$$

hold for all $t \in[0,1]$. Finally, writting the equalities (2.9) and (2.25) in (2.35)

$\left|\frac{p}{b^{p}-a^{p}} \int_{a}^{b} \frac{f(x)}{x^{1-p}} d x-f\left(\left[\frac{a^{p}+b^{p}}{2}\right]^{\frac{1}{p}}\right)\right| \leq M \frac{b^{p}-a^{p}}{4 p\left(\frac{a^{p}+b^{p}}{2}\right)^{1-\frac{1}{p}}} \cdot 2 F_{1}\left(1-\frac{1}{p}, 2 ; 3 ; \frac{a^{p}-b^{p}}{a^{p}+b^{p}}\right)$

Thus, the proof of the Theorem is completed.

Remark 2.1. If we choose $p=1$ in Theorem 2.1, then the results we obtained coincide with the Theorem 1.4.

If we choose $p=-1$ in Theorem 2.1, then we can also give the following corollary.

Corollary 2.1. $\quad f: I \subset(0, \infty) \rightarrow \mathbb{R}$ be an $M$-Lipschitzian function on the interval $I$ of real numbers and $a, b \in I$ with $a<b$. Then, following inequality it holds that:

i) $\quad\left|\frac{f(a)+f(b)}{2}-\frac{a b}{b-a} \int_{a}^{b} \frac{f(x)}{x^{2}} d x\right| \leq M \frac{b-a}{6 a b^{3}}\left[{ }_{2} F_{1}\left(2,2 ; 4 ; 1-\frac{a}{b}\right)+1\right]$,

ii) $\left|\frac{a b}{b-a} \int_{a}^{b} \frac{f(x)}{x^{2}} d x-f\left(\frac{2 a b}{a+b}\right)\right| \leq M \frac{b-a}{2 a b\left(\frac{a+b}{2 a b}\right)^{2}}\left[{ }_{2} F_{1}\left(2,1 ; 3 ; 1-\frac{a}{b}\right)+1\right]$.

By using Theorem 1.2, Lemma 1.1 and Theorem 2.1, we can state the following corollary:

Corollary 2.2. Let the function $f: I \subset(0, \infty) \rightarrow \mathbb{R}$ a differentiable p-convex function on interval $I, a, b \in I$ with $a<b$ and $M=\sup _{t \in[a, b]}\left|f^{\prime}(t)\right|<\infty$. Then:

For $p \geq 1$;

i) $0 \leq \frac{f(a)+f(b)}{2}-\frac{p}{b^{p}-a^{p}} \int_{a}^{b} \frac{f(x)}{x^{1-p}} d x \leq 2 M\left(b^{p}-a^{p}\right)^{\frac{1}{p}} \frac{p^{2}}{(p+1)(2 p+1)}$,

ii) $0 \leq \frac{p}{b^{p}-a^{p}} \int_{a}^{b} \frac{f(x)}{x^{1-p}} d x-f\left(\left[\frac{a^{p}+b^{p}}{2}\right]^{\frac{1}{p}}\right) \leq M\left(\frac{1}{2}\right)^{\frac{1}{p}}\left(b^{p}-a^{p}\right)^{\frac{1}{p}} \frac{p}{p+1}$, 
For $p<0$;

$$
\begin{aligned}
\text { i) } 0 & \leq \frac{f(a)+f(b)}{2}-\frac{p}{b^{p}-a^{p}} \int_{a}^{b} \frac{f(x)}{x^{1-p}} d x \\
& \leq M \frac{b^{p}-a^{p}}{6 p b^{p-1}}\left[{ }_{2} F_{1}\left(1-\frac{1}{p}, 2 ; 4 ; 1-\left(\frac{b}{a}\right)^{p}\right)+1\right] \\
\text { ii) } 0 \leq & \frac{p}{b^{p}-a^{p}} \int_{a}^{b} \frac{f(x)}{x^{1-p}} d x-f\left(\left[\frac{a^{p}+b^{p}}{2}\right]^{\frac{1}{p}}\right) \\
& \leq M \frac{b^{p}-a^{p}}{2 p\left(\frac{a^{p}+b^{p}}{2}\right)^{1-\frac{1}{p}}}\left[{ }_{2} F_{1}\left(1-\frac{1}{p}, 1 ; 3 ; 1-\left(\frac{b}{a}\right)^{p}\right)+1\right] .
\end{aligned}
$$

For $0<p<1$;

i) $0 \leq \frac{f(a)+f(b)}{2}-\frac{p}{b^{p}-a^{p}} \int_{a}^{b} \frac{f(x)}{x^{1-p}} d x \leq M \frac{\left|b^{p}-a^{p}\right|}{3 p} A\left(a^{1-p}, b^{1-p}\right)$,

ii) $0 \leq \frac{p}{b^{p}-a^{p}} \int_{a}^{b} \frac{f(x)}{x^{1-p}} d x-f\left(\left[\frac{a^{p}+b^{p}}{2}\right]^{\frac{1}{p}}\right)$

$$
\leq M \frac{b^{p}-a^{p}}{4 p\left(\frac{a^{p}+b^{p}}{2}\right)^{1-\frac{1}{p}}} \cdot 2 F_{1}\left(1-\frac{1}{p}, 2 ; 3 ; \frac{a^{p}-b^{p}}{a^{p}+b^{p}}\right) .
$$

Remark 2.2. If we choose $p=1$ in Corollary 2.2 , then the results we obtained coincide with the Corollary 1.1.

Proposition 2.1. For $p \geq 1$ and $0<a<b$, the inequalities

$$
\begin{aligned}
\text { i) } 0 & \leq H^{-1}\left(a^{p}, b^{p}\right)-L^{-1}\left(a^{p}, b^{p}\right) \leq \frac{\left(b^{p}-a^{p}\right)^{\frac{1}{p}}}{a^{p+1}} \frac{2 p^{3}}{(p+1)(2 p+1)} \\
\text { ii) } 0 & \leq L^{-1}\left(a^{p}, b^{p}\right)-A^{-1}\left(a^{p}, b^{p}\right) \leq \frac{\left(b^{p}-a^{p}\right)^{\frac{1}{p}}}{a^{p+1}}\left(\frac{1}{2}\right)^{\frac{1}{p}} \frac{p^{2}}{p+1}
\end{aligned}
$$

hold.

Proof. The function $f:(0, \infty) \rightarrow \mathbb{R}, f(x)=x^{-p}(p \geq 1)$ is a differentiable $p$-convex function. By using Corollary 2.2 for $p \geq 1$ :

(i) Since $\left|f^{\prime}(x)\right|=\left|-p x^{-p-1}\right|=p x^{-p-1}$, we obtain

$$
\begin{gathered}
M=\sup _{x \in[a, b]}\left|f^{\prime}(x)\right|=\sup _{x \in[a, b]} p x^{-p-1}=\frac{p}{a^{p+1}}, \\
\frac{f(a)+f(b)}{2}=\frac{a^{-p}+b^{-p}}{2}=\frac{1}{2}\left(\frac{1}{a^{p}}+\frac{1}{b^{p}}\right)=\frac{1}{2}\left(\frac{a^{p}+b^{p}}{(a b)^{p}}\right)
\end{gathered}
$$


and

$$
\int_{a}^{b} \frac{x^{-p}}{x^{1-p}} d x=\int_{a}^{b} \frac{1}{x} d x=\ln b-\ln a
$$

Hence we have

$$
\frac{a^{p}+b^{p}}{2(a b)^{p}}-\frac{p(\ln b-\ln a)}{b^{p}-a^{p}} \leq \frac{\left(b^{p}-a^{p}\right)^{\frac{1}{p}}}{a^{p+1}} \frac{2 p^{3}}{(p+1)(2 p+1)}
$$

(ii) It is easy to see that

$$
\begin{gathered}
f\left(\left[\frac{a^{p}+b^{p}}{2}\right]^{\frac{1}{p}}\right)=\left(\left(\frac{a^{p}+b^{p}}{2}\right)^{\frac{1}{p}}\right)^{-p}=\left(\frac{a^{p}+b^{p}}{2}\right)^{-1}=\frac{2}{a^{p}+b^{p}} \\
\frac{p(\ln b-\ln a)}{b^{p}-a^{p}}-\frac{2}{a^{p}+b^{p}} \leq \frac{\left(b^{p}-a^{p}\right)^{\frac{1}{p}}}{a^{p+1}}\left(\frac{1}{2}\right)^{\frac{1}{p}} \frac{p^{2}}{p+1}
\end{gathered}
$$

Proposition 2.2. For $p \leq-1$ and $0<a<b$, the following inequalities hold:

$$
\text { i) } \begin{aligned}
0 & \leq H^{-1}\left(a^{p}, b^{p}\right)-L^{-1}\left(a^{p}, b^{p}\right) \\
& \leq \frac{\left|b^{p}-a^{p}\right|}{6 a^{p+1} b^{p-1}}\left[{ }_{2} F_{1}\left(1-\frac{1}{p}, 2 ; 4 ; 1-\left(\frac{b}{a}\right)^{p}\right)+1\right],
\end{aligned}
$$

ii) $0 \leq L^{-1}\left(a^{p}, b^{p}\right)-A^{-1}\left(a^{p}, b^{p}\right)$

$$
\leq \frac{\left|b^{p}-a^{p}\right|}{2 a^{p+1}\left(\frac{a^{p}+b^{p}}{2}\right)^{1-\frac{1}{p}}}\left[{ }_{2} F_{1}\left(1-\frac{1}{p}, 1 ; 3 ; 1-\left(\frac{b}{a}\right)^{p}\right)+1\right] \text {. }
$$

Proof. The function $f:(0, \infty) \rightarrow \mathbb{R}, f(x)=x^{-p}(p \leq-1)$ be a differentiable $p$ convex function. By using Corollary 2.2 for $p<0$, we obtain desired inequalities.

Proposition 2.3. For $0<p<1$ and $0<a<b$, the following inequalities hold:

$$
\text { i) } \quad 0 \leq A(a, b)-L_{p-1}^{1-p}(a, b) L_{p}^{p}(a, b) \leq \frac{b^{p}-a^{p}}{3 p} A\left(a^{1-p}, b^{1-p}\right)
$$

ii) $\quad 0 \leq L_{p-1}^{1-p}(a, b) L_{p}^{p}(a, b)-M_{p}(a, b) \leq \frac{b^{p}-a^{p}}{4 p\left(\frac{a^{p}+b^{p}}{2}\right)^{1-\frac{1}{p}}} \cdot{ }_{2} F_{1}\left(1-\frac{1}{p}, 2 ; 3 ; \frac{a^{p}-b^{p}}{a^{p}+b^{p}}\right)$. 
Proof. The function $f:(0, \infty) \rightarrow \mathbb{R}, f(x)=x(0<p<1)$ be a differentiable $p$-convex function. By using the Corollary 2.2 for $p<1$, we can write

$$
\begin{aligned}
\frac{f(a)+f(b)}{2} & =\frac{a+b}{2}, \\
\int_{a}^{b} \frac{x}{x^{1-p}} d x & =\frac{b^{p+1}-a^{p+1}}{p+1}, \\
M & =\sup _{x \in[a, b]}\left|f^{\prime}(x)\right|=1 .
\end{aligned}
$$

So, we obtain followings for Corollary 2.2 (i)-(ii) for $0<p<1$, respectively:

$\left|\frac{a+b}{2}-\frac{p}{b^{p}-a^{p}} \frac{b^{p+1}-a^{p+1}}{p+1}\right|=\frac{a+b}{2}-\frac{p}{b^{p}-a^{p}} \frac{b^{p+1}-a^{p+1}}{p+1} \leq \frac{b^{p}-a^{p}}{3 p} A\left(a^{1-p}, b^{1-p}\right)$

and

$\frac{p}{b^{p}-a^{p}} \frac{b^{p+1}-a^{p+1}}{p+1}-\left(\frac{a^{p}+b^{p}}{2}\right)^{\frac{1}{p}} \leq \frac{b^{p}-a^{p}}{4 p\left(\frac{a^{p}+b^{p}}{2}\right)^{1-\frac{1}{p}}} \cdot{ }_{2} F_{1}\left(1-\frac{1}{p}, 2 ; 3 ; \frac{a^{p}-b^{p}}{a^{p}+b^{p}}\right)$.

\section{Conclusion}

In this paper, using the definition of $M$-Lipschitzian function and some simple mathematical inequalities, we obtained new inequalities related to the right and left sides of the inequality (1.2) for Lipshitz functions. Some results obtained in this study are reduced to the results obtained in [1] in special cases.

\section{REF ER E N C E S}

1. S.S. DRAGOMIR, Y.J. CHO and S.S. KIM: Inequalities of Hadamard's type for Lipschitzian mappings and their applications. Journal of Mathematical Analysis and Applications, 245(2), 489-501, 2000.

2. S.S. DRAGOMIR, C.E.M. PEARCE: Selected Topics on Hermite-Hadamard Inequalities and Its Applications. RGMIA Monograph, 2002.

3. S.S. DRAGOMIR: On Some New inequalities of Hermite-Hadamrd Type for $m$-Convex Functions. Tamkang J. of Math., 33(1), 45-55, 2002.

4. J. HADAMARD: Etude sur les proprietes des fonctions entieres en particulier d'une fonction consideree par Riemann. J. Math. Pures Appl. 58, 171-215, 1893.

5. I. İ̧̧CAN: Hermite-Hadamard type inequalities for harmonically convex functions. Hacettepe Journal of Mathematics and Statistics, 43(6), 935-942, 2014.

6. İ. İŞCAN: New general integral inequalities for Lipschitzian functions via Hadamard fractional integrals, International Journal of Analysis, volume 2014, Article ID 353924, 8., 2014. 
7. İ. İSCAN: Ostrowski type inequalities for p-convex functions, New Trends in Mathematical Sciences, 4(3), 140-150, 2016.

8. İ. İSCAN: Hadamard-type and Bullen-type inequalities for Lipschitzian functions via fractional integrals. Mathematical Sciences and Applications E-Notes, 4(1), 77-87, 2016.

9. İ. İŞCAN, C. ALTUNSOY and M. KADAKAL: Some New Inequalities for Lipschitz Functions via a Functional. GÜFBED/GUSTIJ, 9(2), 301-306, 2019.

10. İ. İSCAN, M. KADAKAL and A. AYDIN: Some new integral inequalities for Lipschitzian functions. An International Journal of Optimization and Control: Theories \& Applications, 8(2), 259-265, 2018.

11. A. KASHURI and R. LIKO: Some $k$-fractional integral inequalities of HermiteHadamard type concerning twice differentiable generalized relative semi- $\left(r ; m, h_{1}, h_{2},\right)$ preinvex mappings. Commun. Optim. Theory 2018, Article ID 6, 2018.

12. A.A. KILBAS, H.M. SRIVASTAVA and J. TRUJILLO: Theory and applications of fractional differential equations. Elsevier, Amsterdam, 2006.

13. M. KUNT and İ. İSCAN: On new Hermite-Hadamard-Fejer type inequalities for $p$ convex functions via fractional integrals. Communication in Mathematical Modeling and Applications, 2(1), 1-15, 2017.

14. M. KUNT and İ. İSCAN: Hermite-Hadamard-Fejer type inequalities for p-convex functions. Arab J. Math. Sci., 23(2), 215-230, 2017.

15. M. KUNT and İ. İ̧̧CAN: Hermite-Hadamard type inequalities for p-convex functions via fractional integrals. Moroccan J. Pure Appl. Anal., 3(1), 22-35, 2017.

16. M. KUNT and İ. İŞCAN: Hermite-Hadamard-Fejer Type Inequalities for p-Convex Functions via Fractional Integrals. Iranian Journal of Science and Technology Transaction A-Science, 42(4), 2079-2089, 2018.

17. M.A. LATIF, S.S. DRAGOMIR and E. MOMANIAT: Some Fejer type integral inequalities for geometrically-arithmetically-convex functions with applications. RGMIA Research Report Collection, 18, Article 25, 18 pp., 2015.

18. C.P. NICULESCU: Convexity according to the geometric mean. Math. Inequal. Appl., 3(2), 155-167, 2000.

19. M.A. NOOR, K.I. NOOR and S. IFTIKHAR: Inequalities via strongly p-harmonic log-convex functions. J. Nonlinear Funct. Anal. 2017, Article ID 20, 2017.

20. M.A. NOOR, K.I. NOOR, F. SAFDAR: Integral inequalities via generalized $(\alpha, m)$ convex functions. J. Nonlinear Funct. Anal. 2017, Article ID 32, 2017.

21. J. PEČARIĆ, J., F. PROSCHAN and Y.T. TONG: Convex Functions, Partial Orderings and Statistical Applications. Academic Press, Inc., 469 pp, Boston, 1992.

22. A.W. ROBERTS and D.E. VARBERG: Convex Functions. Academic Press, 300 pp, New York, 1973.

23. H.M. SRIVASTAVA and D. BANSAL: Close-to-convexity of a certain family of $q$ Mittag-Leffler functions. J. Nonlinear Var. Anal. 1, 61-69, 2017.

24. G.S. YANG and K.L. TSENG: On certain integral inequalities related to HermiteHadamard inequalities. J. Math. Anal. Appl., 239, 180-187, 1999. 
İmdat İşcan

Faculty of Art and Science, Department of Mathematics

Giresun University, 28200, Giresun, Turkey

imdati@yahoo.com

Cuma Altunsoy

Mustafa Kemal Secondary School, Mathematics Teacher

Ministry of Education, Giresun, Turkey

cumaltunsoy@hotmail.com

Huriye Kadakal

Hamdi Bozbağ Anatolian High School, Mathematics Teacher

Ministry of Education, Giresun, Turkey

huriyekadakal@hotmail.com 